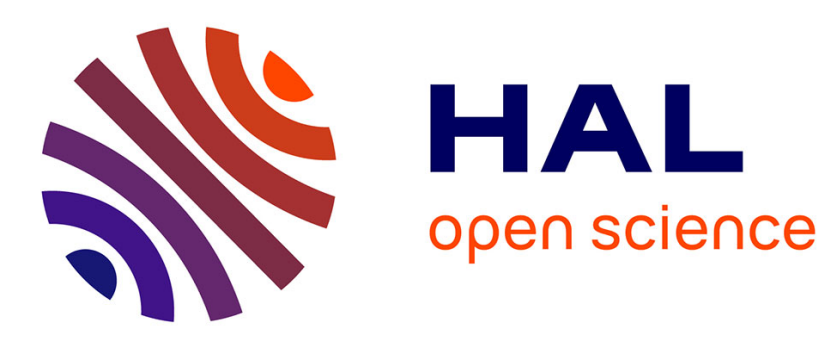

\title{
Beam focusing antenna array technology for non-stationary mobility
}

Hind Zaaraoui, Zwi Altman, Eitan Altman

\section{To cite this version:}

Hind Zaaraoui, Zwi Altman, Eitan Altman. Beam focusing antenna array technology for nonstationary mobility. IEEE Wireless Communications and Networking Conference (WCNC), Apr 2016, Doha, Qatar. hal-01248529

\section{HAL Id: hal-01248529 \\ https://hal.science/hal-01248529}

Submitted on 28 Dec 2015

HAL is a multi-disciplinary open access archive for the deposit and dissemination of scientific research documents, whether they are published or not. The documents may come from teaching and research institutions in France or abroad, or from public or private research centers.
L'archive ouverte pluridisciplinaire HAL, est destinée au dépôt et à la diffusion de documents scientifiques de niveau recherche, publiés ou non, émanant des établissements d'enseignement et de recherche français ou étrangers, des laboratoires publics ou privés. 


\title{
Beam focusing antenna array technology for non-stationary mobility
}

\author{
Hind Zaaraoui*, Zwi Altman* and Eitan Altman ${ }^{\dagger}$ \\ * Orange Labs \\ 38/40 rue du Général Leclerc,92794 Issy-les-Moulineaux \\ Email:\{hind.zaaraoui,zwi.altman\}@orange.com \\ ${ }^{\dagger}$ INRIA Sophia Antipolis, France \\ Email:Eitan.Altman@sophia.inria.fr
}

\begin{abstract}
The aim of this paper $^{\mathrm{a}}$ is to study new antenna array technologies in order to manage efficiently heterogeneous, fixed and mobile traffic. Traffic light close to the cell edge is introduced to generate non stationary mobility pattern in the cell. A car following model is used to model the mobility behavior of the vehicles. A heterogeneous antenna system with different large antenna array technologies is considered: Virtual Small Cell (VSC), virtual small cell with Self-Organizing Network (VSCSON) and beamforming with multilevel global codebook that manages the heterogeneous antenna system at the Base Station (BS). The first two technologies improve the cell performance due to the capability to focus the signal at the traffic concentration near the traffic light. The novel beamforming solution with global codebook can further and significantly improve performance due to the capability to focus the signal along the road and to implicitly balance the traffic between the different antennas. Numerical simulations illustrate the benefits brought about by the different antenna technologies.
\end{abstract}

Index Terms - Vehicular mobility; Multilevel beamforming; Multilevel codebook; Virtual small cell; Self-organizing network; Heterogeneous antenna system; 5G network; $\alpha$-fair utility;

\section{INTRODUCTION}

Large antenna array technology, with beam focusing capabilities has received recently intensive research and development efforts in view of increasing spectral and energy efficiency. This technology is likely to be among the main pillars of $5 \mathrm{G}$ networks in order to achieve the ambitious capacity targets [1].

Three beam focusing technologies are considered here, adapted to environments with significant Line of sight (LoS) component. All three are considered and analyzed in the context of non-stationary mobility. The first technology is the Virtual Small Cell (VSC) or Virtual Sectorization (ViS), ([2]), [3]. It is noted that the term ViS is sometime used when the size of the virtual cell is large with respect to that of a small cell. VSC aims at covering remotely a localized traffic zone, e.g. a hot spot. The VSC is created by a narrow beam generated by an antenna array. It can constitute a cell with a distinct cell ID, and utilize the same frequency resources as the macro-cell (frequency reuse 1). While small cells can be used to cover locally concentrated traffic zone, the associated

\footnotetext{
${ }^{a}$ This work has been partially financed by the ANR IDEFIX project.
}

deployment cost for the backhaul has motivated the study of the VSC technology.

The second technology is the VSC combined with SelfOrganizing Networks (SONs). We consider the VSC and the macro-cell as a single logical cell with a single cell-ID, that dynamically shares frequency resources by means of a SON algorithm [3]. While less resources are allocated to each of the cells (macro- and VSC), the mutual interference between them is suppressed.

Multilevel beamforming has been proposed for backhaul alignment [4] and more recently, for the Radio Access Network (RAN) [5]. The set of multilevel beams defines a codebook with a hierarchical form, from large coverage beams to more focused ones. In [5], the concept of beam planning is proposed, namely the form of the beams is preoptimized to suit the specific need for coverage while taking into account basic geometric parameters of the cell. Multilevel beamforming can be efficiently applied to both Time Division Duplex (TDD) and Frequency Division Duplex (FDD), and is motivated by its capability to handle large codebook size with reduced complexity associated with feedback and processing. The high level focusing allows to minimize the interference generated at neighboring cells.

The third technology is a new generalization of the concept of multilayer codebook. We consider a heterogeneous antenna system of a single BS and one cell. Each antenna can support a single or a set of beams. All the beams of the heterogeneous antenna system comprise a single codebook, denoted here as the global codebook. In this system, the scheduler selects the users to be served according to a utility function (e.g. Proportional Fair (PF)), and then, the best beam from the global codebook is selected iteratively in TDD or FDD systems. The advantage of this solution is the beam selection and resource allocation for the heterogeneous antenna system that is performed at a millisecond time scale, hence allowing to react instantaneously to traffic variations. The load generated by the traffic served using the different antennas is implicitly balanced. Furthermore, the transmissions from the different antennas do not interfere with each other.

The paper analyzes the performance of the above three technologies in the context of non-stationary mobility. The analyzed scenario comprises a macro-cell with fixed users, 
crossed by a road. A traffic light located close to the cell edge generates a non-stationary mobility pattern [6]. Periodic traffic cumulation occurs according to the traffic light periodicity. A simple car following algorithm (see Simulations and Numerical results section) is applied to account for the impact of grouped mobility.

The contributions of the paper are the following:

- Design of a multilevel codebook for serving traffic along a road

- Introduction of the concept of a global codebook of beams for a heterogeneous antenna system of a BS.

- Performance analysis and comparison of beam focusing technologies using antenna arrays for non-stationary mobility pattern

The paper is organized as follows. Section II describes the system-, mobility- and propagation models and explains the utilized scheduling and beam selection algorithm. Section III presents the three beam focusing technologies investigated in the paper, including the VSC, the combination of VSC and $\mathrm{SON}$, and the multilevel beamforming for a heterogeneous antenna system. The performance indicators are formulated in Section IV. The scenario description and the numerical results are described in Section V, followed by concluding remarks in Section VI.

\section{SYSTEM MODEL}

Consider a macro-cell with a BS located at its center. A road crosses the cell with a traffic light close to the cell edge, as shown in Figure 1. The BS has an omni-directional antenna with full cell coverage, and can have one or two additional antennas for the road traffic coverage according to the considered scenario. The reference scenario comprizes an omni-directional macro-cell antenna; The second scenario has an additional VSC antenna covering the area of the red light which is a hotspot like area; The third scenario comprises two antenna arrays with multilevel beamforming capability in addition to the macro-cell antenna (see Figure 4).

\section{A. Traffic model}

Mobile and fixed users are both considered in the cell. Fixed users can be located all over the cell surface (except on the road), while mobile users consist of vehicles moving along a one-way linear road from the left border to the right (Fig.1). All users arrive with an exponential random inter-arrival time with parameters $\lambda_{1}$ and $\lambda_{2}$ that can depend on time:

- vehicular users arrive from the left border of the cell through the road (see Fig.1 with intensity $\lambda_{1}$;

- fixed users arrive uniformly on the cell surface with intensity $\lambda_{2}$.

The traffic light close to the cell edge creates a periodic congestion. In a previous work [6] it has been shown how a heterogeneous network with a small cell and a suitable resource allocation can alleviate congestion and optimize performance. We consider here different approaches, namely
VSC and an antenna array supporting multilevel beamforming as cost efficient alternative solutions which do not require backhaul deployment. While any antenna can serve both fixed and mobile users according to the attachment criterion, the macro-cell antenna serves mainly the fixed users, and the VSC and multilevel beamforming antennas serve mainly users on the road and its direct proximity.

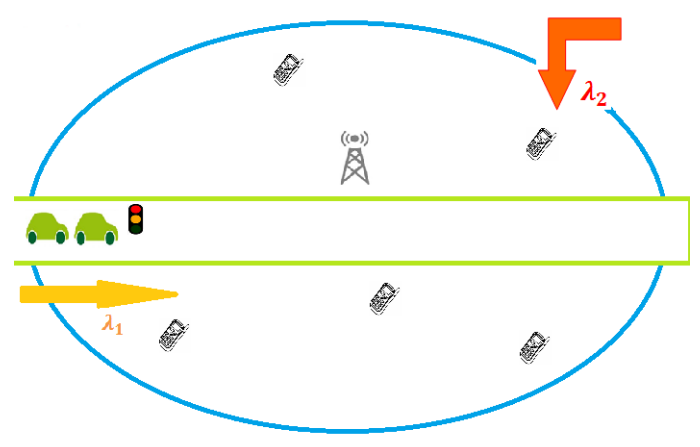

Fig. 1. Macro-cell with a road crossing it. A traffic light is located close to the cell edge. Mobile users enter the cell with intensity $\lambda_{1}$, and fixed users arrive uniformly at the cell area with intensity $\lambda_{2}$.

\section{B. Data rate and scheduling}

Denote by $R\left(x_{i}, t\right)$ the mean data rate of a user located at position $x_{i}$ at time $t . R\left(x_{i}, t\right)$ depends on the considered scheduling, and is computed as the mean instantaneous data rate received during one second (see Fig.2). The PF scheduler is considered throughout the paper, with a time step of $2 \mathrm{~ms}$. A user $K(t)$ is selected by the scheduler if he maximizes a utility function defined as the ratio between its instantaneous and its mean data rates:

$$
K(t)=\operatorname{argmax}_{i \in U \operatorname{sers}} \frac{r\left(x_{i}, t\right)}{R\left(x_{i}, t\right)} .
$$

$r\left(x_{i}, t\right)=\min \left(4.4,0.6 \log _{2}\left(1+\operatorname{SINR}\left(x_{i}, t\right)\right)\right)$ is the instantaneous data rate with modified Shannon formula [7]). $\operatorname{SINR}\left(x_{i}, t\right)$ is the signal to interference plus noise ratio of a user in position $x_{i}$ at time $t$

$$
\operatorname{SINR}_{s}\left(x_{i}, t\right)=\frac{P_{s} h_{s}\left(x_{i}, t\right)}{N_{0}+\sum_{k \neq s} P_{k} h_{k}\left(x_{i}, t\right)} .
$$

$s$ is defined by the best serving cell / beam for a user in position $x_{i}$. The sum in the denominator is taken over the interfering signals from other BSs. $P_{s}$ is the transmitted power and the channel gain $h_{s}$ includes the fading $F$ and the pathloss. $R\left(x_{i}, t\right)$ is calculated as

$$
R\left(x_{i}, t\right)=(1-\epsilon) R\left(x_{i}, t-1\right)+\epsilon 1_{i=K(t)} r\left(x_{i}, t-1\right)
$$

with $\epsilon>0$ being a small averaging parameter. The scheduling algorithm depends on the coherence time of the radio propagation channel which varies with the user speed. In the present work, we assume that the mobile speed does not exceed $50 \mathrm{~km} / \mathrm{h}$ and that the coherence time is large enough for the scheduler to converge in one second, and in this case, the Proportional-Fair scheduler converges to Round-Robin one.

We suppose in this paper symmetric fast fading statistics, namely all the users have the same distribution of fast fading. 


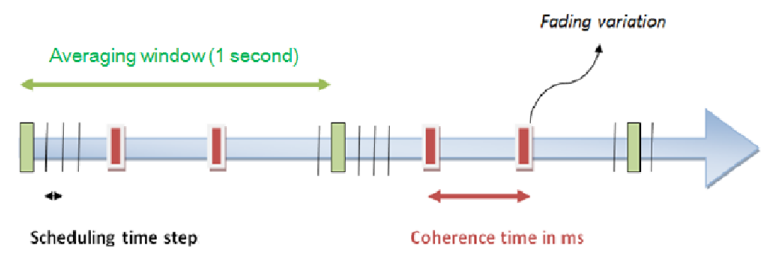

Fig. 2. Scheduling model

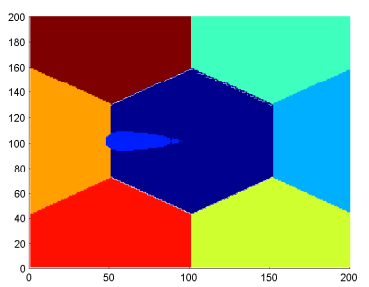

(a) $N_{x} \times N_{z}=6 \times 16$

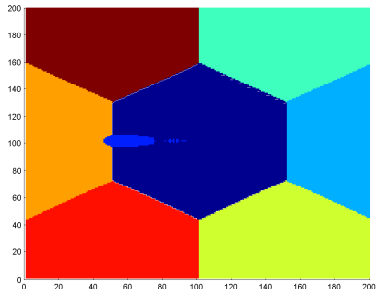

(b) $N_{x} \times N_{z}=24 \times 32$
Fig. 3. VSC antenna array with different array size $N_{x} \times N_{z}$

Two different fast fading distributions are considered: independent Rayleigh fading and Independent Rician fading. The first model corresponds to an environment rich in multipaths due to high density of scatterers. The second one corresponds to an environment with significant direct, LoS component between the transmitter and the receiver. Reference [8] shows that the Rician fading is the appropriate model in the case of opportunistic beamforming (LoS case). To simplify the analytical expression of the fading, we use the approximation of the Rician distribution by the Nakagami model [9] with parameters $m$ and $w$ :

$$
f(x, m, w)=\frac{2 m^{2}}{\Gamma(m) w^{m}} x^{2 m-1} e^{-\frac{m}{w} x^{2}} 1_{x \in] 0, \infty]}
$$

\section{ANTENNA ARRAY BEAM FOCUSING SOLUTIONS}

\section{A. Virtual small cell (VSC)}

A VSC is a remotely created small cell using an antenna array that radiates a narrow beam. The beam is directed towards the desired coverage area using antenna array techniques [3]. The VSC antenna can be located beside the macro-cell BS antenna, thus avoiding backhaul deployment cost as in classical small cells. The VSC beam can either operate on the same frequency bandwidth as the macro-cell (and is denoted here as $V S C$ ) or can dynamically share the bandwidth using a SON algorithm (and is denoted as VSC-SON).

For sake of illustration, consider a $N_{x} \times N_{z}$ antenna array of vertical dipoles, at a distance of $\frac{\lambda}{4}$ from a square metallic conductor, with $\lambda$ being the wavelength. To simplify the model, the reflector is approximated as an infinite Perfect Electric Conductor (PEC). The $N_{x}$ and $N_{z}$ elements in each row and column are equally spaced with distances $d_{x}=0.5 \lambda$ and $d_{z}=0.7 \lambda$, respectively. The full antenna modelling is described in [5], including the antenna tapering which considerably reduces the side lobe level.
While the smaller antenna which supports a wider beam in Figure 3a covers all the road, it is less focused and achieves lower SINR than the the more focused beam in Fig.3b. The latter is designed to mainly cover the area of the traffic light where periodic congestion may occur. The resource allocation for VSC and VSC-SON is described presently.

1) VSC: Full frequency reuse consists of allocating the entire bandwidth to both the macro-cell and the VSC. A fixed power budget $P$ is assumed, namely the BS power is shared equitably between the VSC, $P_{v}$, and the macro-cell antenna, $P_{m}, P / 2=P_{m}=P_{v}$. In this scenario, Significant interference can be expected between the macro-cell and the VSC.

2) VSC-SON: The frequency bandwidth $W$ is shared between the macro-cell and the VSC. A split factor, $\delta$, dynamically optimizes the $\alpha$-fair utility, $U_{\alpha}$, of the users' throughputs [6]. We suppose here that $\alpha=1$, which corresponds to the PF scheduler utility. The objective is to remove the interferences between macro-cell and VSC. Denote by $S_{s}$ and $S_{m}$ the surfaces covered by the VSC and the macro-cell respectively. We want to find a time dependent $\delta$ that optimizes the $\alpha$ fair utility: $U(\delta)=\sum_{x_{i} \in S_{s}} \log \left(\delta R\left(x_{i}, t\right)\right)+\sum_{x_{i} \in S_{m}} \log ((1-$

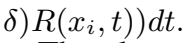

The closed form of the optimal fixed frequency split factor $\delta(t)$ is given as follows [6]:

$$
\delta(t)=\frac{N_{s}(t)}{N_{m}(t)+N_{s}(t)},
$$

where $N_{s}$ the number of users in the VSC and $N_{m}$-in the macro-cell.

\section{B. Multilevel beamforming for heterogeneous antenna system}

Consider a heterogeneous antenna system comprising a macro-cell covering the entire cell and two antenna arrays that are able to follow the users along the road (see Fig.4).

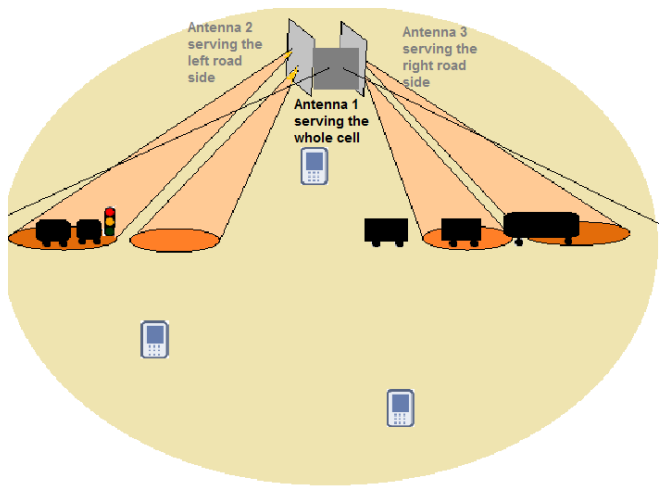

Fig. 4. Two antennas with multilevel beamforming capability cover the traffic along a road crossing the cell

1) Multilevel beamforming: The objective is to focus all the BS power on a user at any position along the road. Each antenna array utilizes a multilevel beam structure that allows to focus the trasmitted signal on users on the road see Figs.5. 


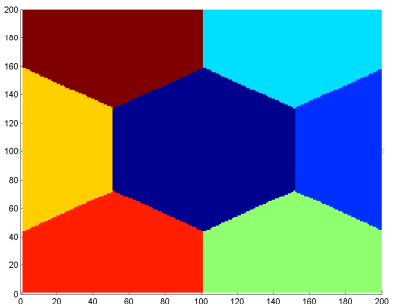

(a) Beam level 0

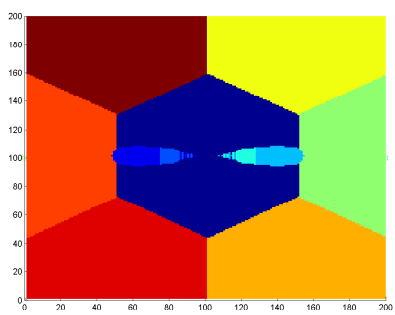

(c) Beams level 2

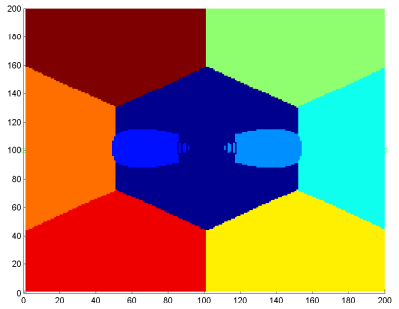

(b) Beams level 1

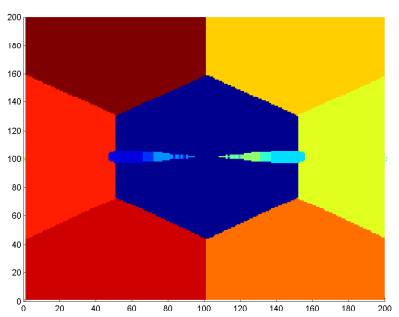

(d) Beams level 3
Fig. 5. Beamforming with global multilevel codebook adapted to the road case

2) Multilevel global codebook: The beamforming with multilevel global codebook for the heterogeneous antenna system is described presently:

- Level 0 (Fig.5a): A global coverage from the central macro-cell antenna (Antenna 1 Fig.4) is used to select the user to be served;

- Level 1 (Fig.5b): Two beams are transmitted by the two parallel antenna arrays (antennas 2 and 3) to cover users on the road on the left and right of the BS;

- Level 2 (Fig.5c): each beam of level 1 is split into two more focused beams to achieve higher rates for vehicular users in the road;

- Level 3 (Fig.5d): beams of level 1 are split into 3 more focused beams than in level 2 .

To choose the user to schedule we use the PF algorithm with the best beam for each user over all the levels. In practice, to reduce complexity, one can design a scheduling algorithm in which the PF utility of a user is calculated for its present beam in level $i$ and its direct neighbors in level $i+1$ and $i-1$ in the multilevel tree of beams. One could think that at any case a user is served with the most focused beam (i.e. at level $3)$. However as $\{$ Beams level i $\} \subseteq\{$ Beams level i- 1$\}$, if a user is between two beams in level 3 it is preferable to take an appropriate beam in a lower level.

\section{Performances IndicAtors}

\section{A. Mean user throughput}

The instantanaeous Mean User Throughput (MUT) is defined as following:

$$
M U T(t)=\frac{1}{N_{t}} \sum_{i=1}^{N_{t}} R\left(x_{i}, t\right)
$$

\section{B. File transfer time}

The File Transfer Time (FTT) for user $i$ is given by :

$$
\begin{array}{r}
F T T_{i}=\min \left\{\int_{0}^{\infty}\left(\left(\frac{d x_{i}(t)}{v_{i}\left(x_{i}(t)\right)} 1_{v_{i} \neq 0}+1_{v_{i}=0}\right) 1_{x_{i}<L}\right) d t,\right. \\
\left.\int_{0}^{\infty} \frac{d \sigma_{i}(t)}{R\left(x_{i}(t)\right)}\right\}
\end{array}
$$

where $x_{i}(t)$ the position of the user $i$ at time $t$ scaning the driving path of length $L$ and that verifies the dynamic equation $\frac{d x_{i}}{d t}=v\left(x_{i}\right) . d \sigma_{i}(t)$ is the volume data that can be transmitted during time $d t(1 \mathrm{sec})$. Denote by $\sigma_{i}(t)$ the volume data left to transfer for user $i$ :

$$
d \sigma_{i}(t)=\min \left\{R\left(x_{i}(t)\right) d t, \sigma_{i}(t)\right\} .
$$

We suppose that the mobile users start their data transfer from the left cell edge while entering in the cell. FTT in mobility (applicable also for fixed users) represents how much time a mobile user needs to transfer all his data as long as he is in the cell.

\section{Throughput outage probability}

In this section we compute the mean Throughput Outage Probability (TOP) of users. This KPI is the probability that the throughput $T h p$ experienced by the users located in a given surface unit does not exceed some threshold $\gamma$. The expression for this probability is $p=P(T h p<\gamma)$. Knowing that the throughput $R(x, t)$ is a bijective function of SINR (Shannon formula supposing that the PF scheduler converges to the Round-Robin one):

$$
p=P(T h p<\gamma)=P\left(S I N R<2^{\frac{\gamma}{W N_{t}}}-1\right) .
$$

We can compute this probability using Monte Carlo and the approximation in [10].

\section{Simulations AND NUMERICAL RESUlTS}

Consider a scenario of a cell with a BS in the center and a road crossing it. We want to serve users with a highest throughput possible. A traffic light is located at the cell edge and creates a periodic congestion. Vehicular users move according to our mobility model defined in the next section. Six technologies scenarios are considered: macro-cell only, four VSC cases with fixed beams equivalent to level 1 and 3 (see Figure (3) and TableIII) and finally the beamforming with mulitevel global codebook.

\section{A. Mobility model}

A deterministic behavior of mobile users is considered in our simulator. We implemente our car following algorithm which preserves their relative order (overtaking is excluded), i.e. $\forall t$ :

$$
x_{i}(t)+v_{i} d t<x_{i-1}(t)+v_{i-1} d t
$$

where $i$ is the $i^{\text {th }}$ car arriving in the cell, $v_{i}$ is its speed and $x_{i}$ - its position. There exists a parameters $a^{\prime}<1$ (see Table II) such that (10) can be reformulated as follows:

$$
v_{i}=a^{\prime}\left(v_{i-1}+\frac{x_{i-1}-x_{i}}{d t}\right)
$$

The car following algorithm is given in Table I. We rewrite the speed of the $i^{t h}$ vehicle $v_{i}(t)=v_{i}\left(x_{i}, t\right)$, and denote by $N_{t}$ the number of users in the cell at time $t$. 
TABLE I

CAR FOLLOWING ALGORITHM

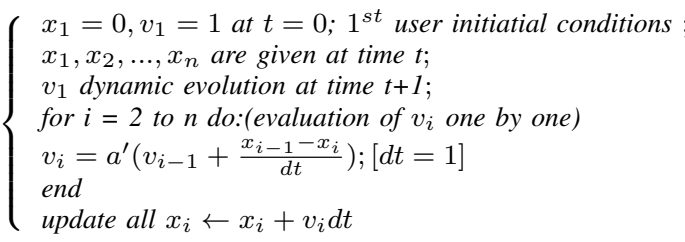

The first vehicle begins its constant deceleration from a safe distance to the traffic light position when the light turns red. The next car follows the behavior of the previous one according to the car following algorithm (Table I). We assume that a vehicle has a limited maximal speed.

TABLE II

NETWORK AND TRAFFIC CHARACTERISTICS

\begin{tabular}{|c|c|}
\hline \multicolumn{2}{|c|}{ Network parameters } \\
\hline Number of macro BSs & 1 \\
\hline Number of interfering BSs & 6 \\
\hline Macro-cell layout & hexagonal trisector \\
\hline Intersite distance & $500 \mathrm{~m}$ \\
\hline Bandwidth & $20 \mathrm{M} \mathrm{Hz}$ \\
\hline Channel characteristics \\
\hline Thermal noise & $-174 \mathrm{dBm} / \mathrm{Hz}$ \\
\hline Macro Path loss (d in km) & $128.1+37.6 \log _{10}(d) \mathrm{dB}$ \\
\hline Data rate averaging parameter $\epsilon$ & 0.2 \\
\hline \multicolumn{2}{|c|}{ Mobility traffic characteristics } \\
\hline$\lambda_{1}$ mobile users & 0.5 users $/ \mathrm{s}$ \\
\hline$\lambda_{2}$ fixed users & 0.5 users $/ \mathrm{s}$ \\
\hline Position traffic light & next to the BS \\
\hline Traffic light periodicity & 200 it. red/ 200it. green \\
\hline Maximal user speed & $5 \mathrm{~m} / \mathrm{it}$. \\
\hline Speed parameter $a^{\prime}$ & 0.8 \\
\hline File size for mobile users $\sigma_{1}$ & $80 \mathrm{Mbits}$ \\
\hline File size for fixed users $\sigma_{2}$ & $1 \mathrm{Mbits}$ \\
\hline
\end{tabular}

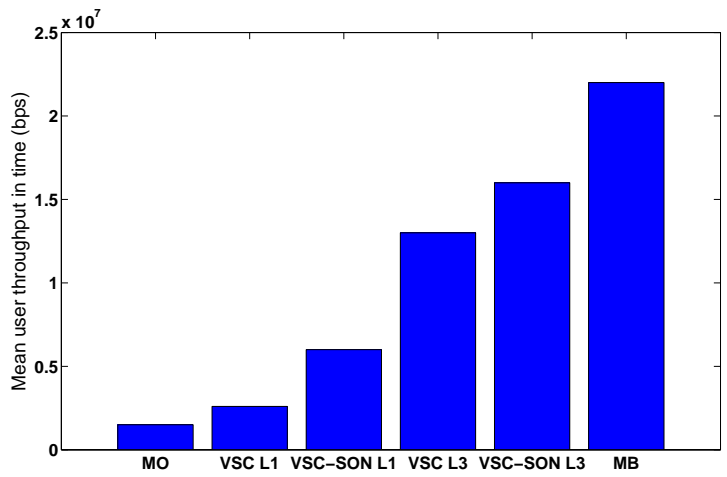

Fig. 6. Mean user throughput in time for the six scenarios: Macro only (MO), VSC in full reuse level 1 and level 3 cases(VSC L1 and VSC L3), VSCSON level 1 and level 3 cases (VSC-SON L1 and VSC-SON L3), multilevel beamforming $(\mathrm{MB})$

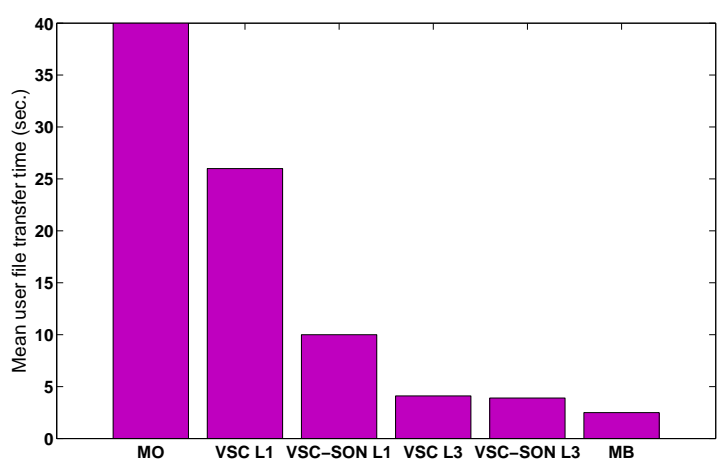

Fig. 7. Mean file transfer time for the six scenarios: Macro only (MO), VSC in full reuse level 1 and level 3 cases(VSC L1 and VSC L3), VSC-SON level 1 and level 3 cases (VSC-SON L1 and VSC-SON L3), multilevel beamforming (MB)

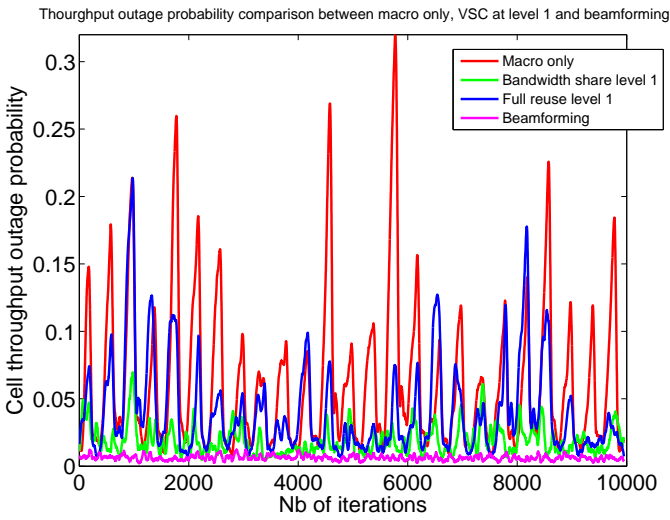

Fig. 8. Throughput outage probability for macro-cell only (Macro only), VSC in level 1(Full reuse level 1), VSC-SON in level 1(Bandwidth share level 1) and multilevel beamforming (Beamforming) scenarios

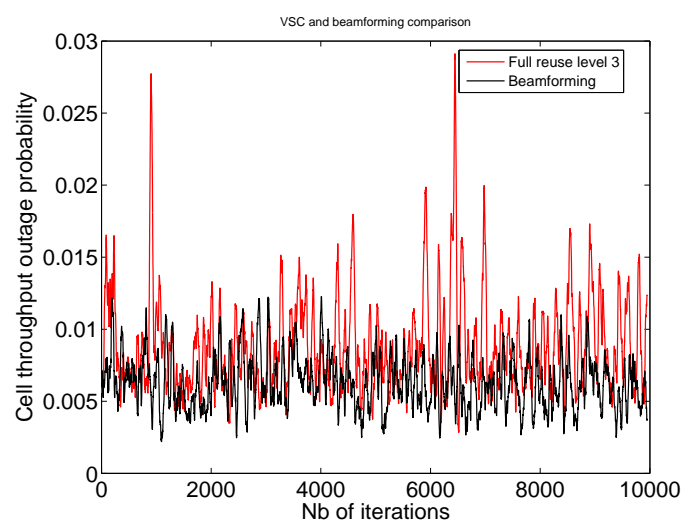

Fig. 9. Throughput outage probability for VSC in level 3 (Full reuse level 3 ) and multilevel beamforming (Beamforming) scenarios

\section{B. Technologies performance comparison}

In this section, we compare the six scenarios cited above. The group of users cumulated by the red light will end up leaving the VSC coverage and therefore another antenna 
system is needed to follow the vehicular users in the cell. Multilevel beamforming with global codebook could be an effective solution. The performance indicators covering the different cases are depicted in Figures 6 for the mean MUT, 8-9 for mean throughput outage probability and in Figure 7) for the mean FTT. One can see that the performance of the multilevel beamforming technology outperforms the VSC technology.

TABLE III

CHARACTERISTICS OF VSC BEAMS LEVEL

\begin{tabular}{|c|c|c|c|}
\hline Beams level & Nx & Nz & Antenna angle \\
\hline Level 0 & 0 & 14 & 10 \\
\hline Level 1 & 6 & 16 & 12 \\
\hline Level 2 & 12 & 16 & 12.5 \\
\hline Level 3 & 24 & 32 & 9.5 \\
\hline
\end{tabular}

The first point observed in the TOP curves (Fig.8 and Fig.9) is that for the case of macro only, as users are periodically accumulated in the traffic light at the cell edge, their data rate condition gets worst with the increased traffic congestion. The departure rate of users from the network decreases and the throughput gets lower which induces a sharp increase in the TOP as shown in Fig.(8). We also notice that for the 6 cases the periodic behaviour of the curves is reduced as the degree of beam focusing is increased. To alleviate the impact of the congestion due to the red light, VSC and multilevel beamforming are investigated:

- VSC with beam level 1: full reuse and bandwidth sharing are both considered. In this case, the bandwidth sharing scenario is three times better than the full reuse one (see Fig.6). The periodicity is almost lost in the bandwidth sharing (VSC-SON) case as departure rate of users is getting higher (better data rate is achieved). Moreover, the more the VSC beam is narrowed the higher are the cell and users performance.

- VSC with beam level 3: For the case of the narrowest beam (level 3), VSC and VSC-SON achieve similar MUT but differ in the FTT. The impact of the macrocell interference on the SINR becomes insignificant as the beam gets narrower: in Fig.6 MUT in VSC-SON is only $10 \%$ higher than that of the VSC MUT in level 3 comparing to three times at level 1.

- Beamforming with multilevel global codebook: one might expect that the radio performances of the cell for the multilevel beamforming would be the same as the VSC as it is the same beam focalised at the traffic light location for both cases. What makes the difference between these two cases is that vehicular users will leave the small cell as soon as the traffic light turns green. The impact of users leaving the VSC is shown through the high peaks in TOP: probability varying between $1.3 \%$ and $3 \%$ for the full reuse in beam level 3 (see Fig.9). These peaks are not anymore periodic in these cases as the departure of users from the network becomes higher as data rate is bigger. The outage probability peaks disappear in the beamforming scenario (see Fig.9).
The mean TOP in this case equals $0.7 \%$. Users who leave the VSC have a significant impact on the network performance: MUT for multilevel beamforming is $40 \%$ higher than that of of the best VSC scenario i.e. with bandwidth sharing at level 3 (Fig.6).

\section{CONCLUSION}

This paper has considered three large antenna array technologies to efficiently manage fixed and mobile users: VSC, VSC-SON and beamforming with multilevel global codebook. We have shown how to adapt the concept of beam planning to construct a multilayer codebook for serving traffic along a road. The beams are designed a priori to fit the coverage needs along the traffic axis.It is shown that due to mobility, the beamforming with multilevel global codebook provides a significant performance in FTT, MUT and TOP with respect to the two VSC technologies. The better performance of the multilayer beamforming with global codebook is two folds: (i) additional focused beams are added which cover all the road, and (ii) the ressource allocation between the three antennas is made almost at real time which implicitly balances the traffic between all the antennas.

\section{REFERENCES}

[1] A. Osseiran et al., "Scenarios for 5G mobile and wireless communications: the vision of the METIS project," IEEE Communications Magazine, vol. 52, no. 5, pp. 26-35, 2014.

[2] A. Galindo-Serrano, S. Martinez Lopez, and A. Gati, "Virtual small cells using large antenna arrays as an alternative to classical HetNets," in First International Workshop on Intelligent Design and Performance Evaluation of LTE-Advanced Networks, Glasgow, Scotland, May 2015.

[3] A. Tall, Z. Altman, and E. Altman, "Virtual sectorization: design and self-optimization," in 5th International Workshop on Self-Organizing Networks (IWSON 2015), Glasgow, Scotland, May 2015.

[4] S. Hur et al., "Millimeter wave beamforming for wireless backhaul and access in small cell networks," IEEE Transactions on Communications, vol. 61, no. 10, pp. 4391-4403, 2013.

[5] A. Tall, Z. Altman, and E. Altman, "Multilevel beamforming for high data rate communication in 5G networks," arXiv preprint arXiv:1504.00280, 2015.

[6] H. Zaaraoui and Z. Altman, "Alleviating cellular network congestion caused by traffic lights," in Proc. IEEE Vehicular Technology Conference (VTC Spring), 2015. IEEE, 2015, pp. 1-5.

[7] 3GPP, "Evolved Universal Terrestrial Radio Access (E-UTRA); Radio Frequency (RF) system scenarios," 3rd Generation Partnership Project (3GPP), TR 36.942, Sep. 2012.

[8] P. Viswanath, D. N. Tse, and R. Laroia, "Opportunistic beamforming using dumb antennas," IEEE Transactions on Information Theory, vol. 48, no. 6, pp. 1277-1294, 2002.

[9] U. Charash, "Reception through nakagami fading multipath channels with random delays," IEEE Transactions on Communications, vol. 27, no. 4, pp. 657-670, 1979.

[10] Y. Zhao, R. Adve, and T. J. Lim, "Outage probability at arbitrary SNR with cooperative diversity," IEEE communications letters, vol. 9, no. 8, pp. 700-702, 2005. 